\title{
Doppelperfekt und Plusquamperfekt im Hoch- und Oberrheinalemannischen Südwestdeutschlands*
}

\author{
Jens Leonhard (Freiburg i. Br.)
}

\begin{abstract}
In this paper I discuss the tenses Doppelperfekt and Plusquamperfekt in spoken High Alemannic and Upper-Rhine Alemannic in south-western Germany with a usage-based approach and a statistical evaluation. I show that both Doppelperfekt and Plusquamperfekt have a past perfect meaning, i. e. they refer to a point of time in the past placed before another point of time in the past. Furthermore, I account for a language change in the timespan from 1974 to 2013 in which the Plusquamperfekt becomes more frequent in relation to the Doppelperfekt. Additionally, I show that speakers use the Doppelperfekt in the old data with both auxiliaries (sein and haben), and in the new data only with the auxiliary haben. In contrast to this, the Plusquamperfekt is used in the old data only with the auxiliary sein, and in the new data with both auxiliaries sein and haben. These results could lead to the conclusion that the Doppelperfekt und Plusquamperfekt cannot exist simultaneously in an equivalent manner.
\end{abstract}

\section{$1 \quad$ Einleitung}

Ich untersuche in diesem Beitrag das Doppelperfekt im Hoch- und Oberrheinalemannischen Südwestdeutschlands qualitativ und quantitativ. Als Doppelperfekt werden alle Konstruktionen betrachtet, die das einfache Perfekt durch das Partizip des jeweiligen Auxiliars (also gehabt oder gewesen) erweitern (cf. Rödel 2007: 13), wie z. B. in:

a. Sie hat die Arbeit gemacht gehabt.

b. Er ist gekommen gewesen.

Im Bereich des Doppelperfekts sah Hennig (2000: 196) die größten Defizite in der Tempusforschung. Dort fehlten insbesondere quantitative Untersuchungen mit statistischer Auswertung und Untersuchungen des Doppelperfekts in Dialekten. In den letzten Jahren sind einige neuere Arbeiten zum Doppelperfekt im Deutschen entstanden (u. a. Hundt 2011, BuchwaldWargenau 2012, Zybatow 2015, Brandner et al. 2016, Haß 2016 und Zybatow/Weskott 2018).

\footnotetext{
* Ich danke Tobias Streck für seine Hilfe bei den Transkriptionen und bei kleineren und größeren Fragen. Außerdem danke ich Peter Auer und den beiden anonymen GutachterInnen für hilfreiche Kommentare zu diesem Beitrag.
} 
Insbesondere ist hier Rödels umfassende und quantitative Arbeit zum Doppelperfekt im Deutschen (2007) zu nennen. In dieser vergleicht er das Doppelperfekt mit dem einfachen Perfekt im gesamten deutschen Sprachgebiet (cf. Rödel 2007: 57).

Viele Arbeiten, die sich mit dem Doppelperfekt befassen, ziehen Vergleiche zum Plusquamperfekt (cf. Behaghel 1924, Breuer/Dorow 1996, Maiwald 2002, Buchwald 2005, Rödel 2007 und Brandner et al. 2016). Gerade in älteren Arbeiten wird meist die These vertreten, das Doppelperfekt sei ein Ersatz für das im Zuge des oberdeutschen Präteritumschwunds ausgefallene Plusquamperfekt (u. a. bei Ölinger 1574, Schottel 1641, Aichinger 1754, Sütterlin 1900, Behaghel 1924, Lindgren 1963, Trier 1965, Erben 1980, Semenjuk 1981, Gersbach 1982 und König 1996). ${ }^{1}$ Die Notwendigkeit eines Plusquamperfekt-Ersatzes erscheint allerdings aus zwei Gründen fraglich. Zum einen koexistieren in vielen Gebieten Doppelperfekt- und Plusquamperfekt-Formen, weshalb ein Plusquamperfekt-Ersatz unplausibel erscheint (cf. dazu Litvinov 1969: 18-20, Şandor 2002: 267, Rödel 2007: 86, Hundt 2011: 12 und Buchwald-Wargenau 2012: 52). Zum anderen trat das Doppelperfekt schon vor dem endgültigen Vollzug des Präteritumschwunds auf. Das Doppelperfekt kann also nicht in Folge des Präteritumschwunds entstanden sein. Litvinov und Radčenko (1998: 92) führen hierzu Literaturbelege aus dem 15. Jahrhundert an. Rödel (2007: 191f.) bemerkt, dass zu diesem Zeitpunkt der Präteritumschwund zwar bereits begonnen hatte, aber das Präteritum keineswegs in entscheidender Weise auf dem Rückzug war. Das Doppelperfekt scheint also nicht als Ersatz für das entfallene Plusquamperfekt entstanden zu sein, sondern existierte bereits davor. Dennoch stellt sich die Frage nach dem funktionellen Unterschied von Doppelperfekt und Plusquamperfekt.

Ausgehend von der Theorie des Plusquamperfekt-Ersatzes bietet es sich für das heutige Alemannisch an zu untersuchen, ob Doppelperfekt und Plusquamperfekt mit dem Ausdruck von Vorvergangenheit dieselbe Bedeutung haben. Sollten die beiden koexistierenden Tempora bedeutungsgleich oder -ähnlich sein, wäre denkbar, dass sie Gegenstand eines momentanen Sprachwandels im Alemannischen Südwestdeutschlands sind. Ein solcher Sprachwandel könnte Rückschlüsse auf das Zusammenwirken der beiden Tempora zulassen und damit auf einen möglichen Zusammenhang zwischen Verbreitung des Doppelperfekts und Verschwinden des Plusquamperfekts. Zusätzlich untersuche ich, ob Doppelperfekt und Plusquamperfekt mit beiden Hilfsverben gebildet werden können und ob diachron Bildungsbeschränkungen zu sehen sind. Daher vergleiche ich die Verteilung der haben- und der sein-selegierenden Verben bei beiden Tempora synchron und diachron.

Das Alemannische Südwestdeutschlands ist als Untersuchungsgebiet aus zwei Gründen geeignet. Zum einen koexistieren dort - wie ich in diesem Beitrag noch zeigen werde - die beiden Tempora Doppelperfekt und Plusquamperfekt. ${ }^{2}$ Zum anderen ist die jüngste Entwicklung im Alemannischen Südwestdeutschlands durch die Erhebungen des Südwestdeutschen Sprachatlasses (Steger/Schupp 1993) und der Projekte REDI (Stoeckle 2014 und Hansen-Morath 2016)

\footnotetext{
${ }^{1}$ Durch den Verlust des Präteritums konnten auch die Präteritalformen der Vollverben haben und sein, die zur Bildung des Plusquamperfekts notwendig sind, nicht mehr gebildet werden (Rödel 2007: 196).

${ }^{2}$ Im Alemannischen der Schweiz dagegen ist der Indikativ Präteritum und dadurch auch das Plusquamperfekt völlig verschwunden (cf. Jörg 1976: 183 und Rowley 1983: 163). Ein Konjunktiv Präteritum lässt sich allerdings nachweisen (cf. Wilde 2015: 245).
} 
und FLARS (Auer et al. 2015) sehr gut dokumentiert. Die dort erhobenen spontansprachlichen Daten erlauben einen qualitativen und quantitativen Blick auf das Verhältnis von Doppelperfekt und Plusquamperfekt.

Ich konzentriere mich in diesem Aufsatz auf Indikativ-Formen. Das hat zwei Gründe. Zum einen verhalten sich Konjunktiv-Formen grundsätzlich anders als Indikativ-Formen und müssen daher gesondert betrachtet werden (cf. Thieroff 1992: 220-222). Zum anderen ist ein Vergleich von Doppelperfekt und Plusquamperfekt nur dann möglich, wenn ausschließlich Fälle betrachtet werden, in denen die beiden Tempora miteinander konkurrieren könnten. Da es im Deutschen allerdings keinen Konjunktiv I des Plusquamperfekts gibt, wird in diesen Fällen obligatorisch das Doppelperfekt als Ersatzform gewählt (cf. Thiel 1964: 85). Hundt untersuchte die schriftlichen Korpora „Archiv der geschriebenen Sprache“ des IDS, das DWDS ${ }^{3}$ und ein Korpus aus Artikeln der ZEIT (cf. Hundt 2011: 3) und stellte dabei fest, dass mehr als die Hälfte aller Doppelperfekt-Belege im Konjunktiv steht (siehe ibd.: 23). Ich erfasse daher die Konjunktiv-Formen in meinen Korpora, um das Ergebnis von Hundt (2011) in der gesprochenen Sprache des Hoch- und Oberrheinalemannischen zu überprüfen.

Um die Frage nach einem Sprachwandel beantworten zu können, vergleiche ich zwei Korpora aus den Jahren 1974-1985 bzw. 2007-2013 miteinander und überprüfe, ob sich die Frequenz des Plusquamperfekts und des Doppelperfekts verändert hat.

Zunächst stelle ich die beiden Korpora vor, die dieser Arbeit zugrunde liegen. Im Anschluss daran untersuche ich anhand der spontansprachlichen Daten der Korpora, ob Doppelperfekt und Plusquamperfekt zeitliche Vorvergangenheit ausdrücken. Daraufhin betrachte ich die diachrone Entwicklung von Doppelperfekt und Plusquamperfekt unter Berücksichtigung der Hilfsverben, die zur Tempusbildung gebraucht werden. Abschließend werde ich die Frage diskutieren, welche Schlussfolgerungen sich daraus für das Zusammenwirken von Doppelperfekt und Plusquamperfekt im Hoch- und Oberrheinalemannischen ergeben.

\section{Korpus und Methode}

Die Grundlage der Untersuchung bilden zwei Korpora, die ich aus den Erhebungen des Südwestdeutschen Sprachatlasses (SSA) sowie aus den Erhebungen der Projekte REDI (Stoeckle 2014 und Hansen-Morath 2016) und FLARS (Auer et al. 2015) zusammengestellt habe. Die Aufnahmen entstanden in den Jahren 1974-1985 bzw. 2007-2013. Die Korpora decken also einen Untersuchungszeitraum von 39 Jahren ab.

Das erste Korpus setzt sich aus Aufnahmen des SSA zusammen. Der SSA umfasst insgesamt 579 Erhebungsorte, die in einem durchschnittlichen Abstand von 6,4 km über die ehemaligen Regierungsbezirke Südbaden und Südwürttemberg verteilt sind (cf. Steger/Schupp 1993: 4). Die Gewährspersonen waren über 60 Jahre alt, im Ort geboren und aufgewachsen (siehe ibd.: 34). Auch deren Eltern sollten aus demselben Ort stammen. Pro Erhebungsort wurden mindestens zwei Gewährspersonen ausgesucht (eine Frau und ein Mann (ibd.)). In einer Teilmenge

\footnotetext{
${ }^{3}$ Dabei handelt es sich um das Korpus Digitales Wörterbuch der deutschen Sprache der Berlin-Brandenburgischen Akademie der Wissenschaften. Dieses enthält verschiedene Textsorten, darunter diverse Wörterbücher sowie Texte aus Zeitungen, Zeitschriften und Blogs.
} 
der Erhebungsorte wurden Tonaufnahmen, die ursprünglich für eine spätere Kontrolle der Transkription etc. gedacht waren, angefertigt. Die Aufnahmen dokumentieren teilweise Ausschnitte der Erhebungssituation, jedoch auch Konversationen unter Verwendung von Fragen ohne vorgegebene Fragestellung (cf. Steger/Schupp 1993: 35). Für meine Untersuchung habe ich lediglich diese spontansprachlichen Teile und die Ortspunkte herangezogen, die im Hoch- und Oberrheinalemannischen bzw. im fränkisch-schwäbischen Grenzgebiet liegen, um eine Vergleichbarkeit mit dem zweiten, jüngeren Korpus zu gewährleisten. Daraus ergeben sich für meine Untersuchung 84 Orte.

Das zweite Korpus fußt auf den Erhebungen der Projekte REDI (2014) und FLARS (2015). Für das Korpus REDI (2014) wurden Untersuchungsorte im alemannischen Dreiländereck (Deutschland, Frankreich, Schweiz) ausgewählt (Stoeckle 2014: 99); für das Projekt FLARS (2015) wurde der gesamte Grenzverlauf des Hoch- und Oberrheinalemannischen im links- und rechtsrheinischen Gebiet untersucht (Auer et al. 2015: 326). Für meinen Beitrag habe ich die Erhebungsorte herangezogen, die im deutschen Staatsgebiet liegen. Somit enthält mein Untersuchungskorpus REDI+FLARS 46 Ortspunkte. Diese Ortspunkte sind gleichmäßig über das Hoch- und Oberrheinalemannische Südwestdeutschlands verteilt. Darüber hinaus handelt es sich bei diesen Orten (mit zwei minimalen Abweichungen) ${ }^{4}$ um Orte, die bereits im Südwestdeutschen Sprachatlas untersucht wurden (cf. Stoeckle 2014: 99f.), um einen historischen Vergleich zu ermöglichen (cf. Auer et al. 2015: 327).

In den beiden neueren Projekten (REDI und FLARS) gibt es jeweils eine jüngere und eine ältere Gruppe an Gewährspersonen (Stoeckle 2014: 102f.). Aus diesen beiden Erhebungen habe ich die älteren Gruppen ausgewählt, also diejenigen Gewährspersonen, die zum Zeitpunkt der Erhebung zwischen 60 und 70 Jahre alt waren. Für jeden Erhebungsort ergaben sich dadurch mindestens vier Gewährspersonen (zwei Frauen und zwei Männer). Ich habe die Auswahl für mein Untersuchungskorpus auf diese ältere Gruppe an Gewährspersonen konzentriert, da laut Mattheier (1994: 427) mit dem Eintritt ins Rentenalter eine Zuwendung zu informelleren Sprechweisen und damit auch zum Dialekt hin geschieht (cf. auch Stoeckle 2014: 103). Somit können mögliche diachrone Unterschiede in der Verwendung von Doppelperfekt und Plusquamperfekt auf einen Sprachwandel des Alemannischen Südwestdeutschlands zurückgeführt werden und nicht auf eine Annäherung der Sprecher an den Standard.

Die Informantinnen und Informanten sind am jeweiligen Erhebungsort geboren, beide Elternteile kommen aus dem alemannischen Sprachgebiet Deutschlands (cf. Auer et al. 2015: 328). Durch 40 Übersetzungssätze wurde die Dialektkompetenz der Gewährspersonen getestet (cf. ibd.). Im Anschluss daran führten die Exploratorinnen und Exploratoren (teilweise alleine, teilweise zu zweit) mit den Gewährspersonen ethnodialektologische Befragungen durch und stellten Fragen zu Sozialdaten und Sprachgebrauch der Informantinnen und Informanten (cf. Stoeckle 2014: 104). In der vorliegenden Untersuchung habe ich nur die freien Gespräche be-

\footnotetext{
${ }^{4}$ Anstelle der Gemeinde Grunern wurde für FLARS (2015) das direkt angrenzende Staufen und anstelle der Freiburger Stadtteile Herdern und St. Georgen wurden die Stadtteile Betzenhausen und Lehen gewählt (Stoeckle 2014: 100).
} 
rücksichtigt. Die Interviews hatten eher informellen Charakter über Dialekte, sodass die ethnodialektologischen Fragen paraphrasiert wurden (cf. Stoeckle 2014: 105). Dadurch entstanden viele freie Gespräche und spontansprachliche Daten.

\section{$3 \quad$ Vorvergangenheitsbedeutung von Doppelperfekt und Plusquamperfekt}

\subsection{Das Reichenbach'sche Tempussystem}

Die Hauptfunktion von Tempus ist der zeitliche Verweis von einer Zeitstufe auf eine andere. Zur Verdeutlichung der deiktischen Funktion des Tempus möchte ich das Reichenbach'sche System vorstellen. Nach Reichenbach (1947: 273) lassen sich drei Zeitpunkte unterscheiden, die auf einer von der Vergangenheit über die Gegenwart in die Zukunft laufenden Zeitachse liegen: den Sprech[zeit]punkt, den Ereignis[zeit]punkt und den Referenz[zeit]punkt. Häufig finden sich in der Literatur anstelle von Zeitpunkten auch Begrifflichkeiten wie Zeitintervalle (cf. hierzu u. a. Ehrich/Vater 1989: 119). Dies wird dadurch begründet, dass grundsätzlich jede beschriebene Handlung, egal ob punktuell oder durativ, eine zeitliche Ausdehnung besitzt. Ich bezeichne in dieser Arbeit die in dieser Hinsicht undifferenzierten Reichenbach'schen Zeitpunkte als Sprechzeit, Ereigniszeit und Referenzzeit.

Unter Sprechzeit verstehe ich mit Reichenbach (1947: 273) die Zeit, zu der eine Äußerung getätigt wird. Diese ist nicht mit der Gegenwart gleichzusetzen, da sie nicht in allen Fällen identisch zur Gegenwart ist. Als Ereigniszeit betrachtet Reichenbach den Zeitpunkt, zu dem das beschriebene Ereignis (also die Verbalhandlung) tatsächlich stattfindet (cf. ibd.). Die Referenzzeit beschreibt einen Punkt, der relativ zu den beiden anderen Zeiten gesetzt werden kann (cf. ibd.). Er kann also vor, nach oder zwischen den anderen beiden Zeiten liegen oder mit einer der beiden zusammenfallen. Während die Ereigniszeit also den objektiven Zeitpunkt eines Ereignisses angibt, verweist die Referenzzeit auf einen vom Sprecher subjektiv festgelegten, i. e. frei wählbaren, Zeitpunkt, von dem die Ereigniszeit aus betrachtet wird. Die Referenzzeit ist besonders zur Beschreibung der Verweisfunktion eines Tempus der Vorvergangenheit (wie dem Plusquamperfekt im Standarddeutschen) notwendig.

„Melanie kam um zwei Uhr bei Max vorbei, aber Max war um zwei Uhr bereits gegangen.“

Der Bedeutungsunterschied dieser beiden Teilsätze lässt sich mit der Distinktion Sprechzeit/Ereigniszeit alleine nicht erklären. Die Ereigniszeit beider beschriebener Ereignisse liegt (vereinfacht gesagt) in der Vergangenheit, die Sprechzeit in der Gegenwart. Erst durch das Hinzuziehen eines weiteren Zeitpunktes kann ein Bedeutungsunterschied festgemacht werden. So gibt die Präpositionalphrase ,um zwei Uhr“ in beiden Sätzen nicht die Ereigniszeit an, sondern die Referenzzeit. Während diese im ersten Teilsatz zeitgleich zur Ereigniszeit zu situieren ist, liegt sie im zweiten Teilsatz zeitlich nach der Ereigniszeit. Im einen Fall kommt Melanie also um zwei Uhr vorbei, im anderen ist Max zu irgendeiner Zeit vor zwei Uhr gegangen. Demzufolge ergibt sich für den ersten Teilsatz folgende Anordnung der Zeiten: Ereigniszeit = Referenzzeit (,um zwei Uhr') > Sprechzeit (,zeitlich nach zwei Uhr'); für den zweiten Teilsatz folgende Anordnung: Ereigniszeit (,zeitlich vor zwei Uhr') > Referenzzeit (,um zwei Uhr') > Sprechzeit (,zeitlich nach zwei Uhr'). 


\subsection{Indizien zur Identifizierung der Vorvergangenheitsbedeutung}

Ich schreibe einem Tempus Vorvergangenheitsbedeutung zu, wenn es eine Referenzzeit setzt, die in der Vergangenheit liegt und auf eine dazu zeitlich frühere Ereigniszeit verweist. Eine eindeutig vorvergangene Lesart lässt sich beim Großteil der Doppelperfekt- und Plusquamperfekt-Belege nachweisen. Hinreichende Kriterien dafür sind vor allem Subjunktionen und Adverbien, aber auch Logik und Weltwissen. Dabei kann entweder die Vor-Vorzeitigkeitsbedeutung von Doppelperfekt und Plusquamperfekt ausgedrückt werden oder die Nachzeitigkeitsbedeutung des Kontrast-Tempus. Für Adverbien bedeutet das beispielsweise, dass sowohl die Verbindung aus scho(n) und Doppelperfekt/Plusquamperfekt als auch die Verbindung aus no (,dann') und kontrastierendem Perfekt auf eine Vorvergangenheitsbedeutung von Doppelperfekt oder Plusquamperfekt hindeuten.

Temporale Subjunktionen, die auf eine Vorzeitigkeitsbedeutung von Doppelperfekt und Plusquamperfekt hindeuten (wie standarddeutsch nachdem), kommen in den Untersuchungskorpora nicht vor. Für die Subjunktion nachdem gibt es im Badischen Wörterbuch ${ }^{5}$ nur vereinzelte Belege aus dem frühen 15. Jahrhundert (cf. BW: Band 4: 3). Andere temporale Subjunktionen mit Vorzeitigkeitsbedeutung werden im Badischen Wörterbuch nicht genannt. Stattdessen wird für eine Vorzeitigkeitsbedeutung auf das Adverb darnach verwiesen (cf. BW: Band 1: 426f.). Anstelle eindeutiger temporaler Subjunktionen werden im Alemannischen die temporalen Subjunktionen wenn und wo gebraucht. Diese können aber je nach Kontext auf Gleich- oder auf Vorzeitigkeit hindeuten und daher nicht als Indikatoren für eine Vorvergangenheitsbedeutung dienen. Ich werde weiter unten auf diese Subjunktionen eingehen.

Neben den eben aufgeführten temporalen Subjunktionen mit Vorzeitigkeitsbedeutung können auch kausale und konzessive Subjunktionen die Vorvergangenheitsbedeutung von Doppelperfekt und Plusquamperfekt indizieren. Im Untersuchungskorpus war die kausale Subjunktion weil zu finden. Anders als entsprechende temporale Subjunktionen drückt die Subjunktion weil zwar nicht per se Vorzeitigkeit aus, dennoch kann auch weil als Indikator für Vorzeitigkeit dienen. Das ist der Fall, wenn eine Ursache-Wirkungs-Beziehung im Sinne einer HandlungFolgehandlung ausgedrückt wird. Demnach ist die durch die Subjunktion weil eingeleitete ursächliche Handlung immer vorzeitig zu deren Wirkung. Es lässt sich beispielsweise in folgendem Satz auch ohne eigentliche Vorzeitigkeitsmarkierung eine Vorzeitigkeit erkennen: „Wir hatten Brot im Haus, weil ich es geholt hatte."

Beispiel (1) ist dem Korpus SSA entnommen. Hier berichtet die Sprecherin GP2_347 von ihrer Zeit als Hebamme.

(1) Krankenhaus (Loffenau: SSA)

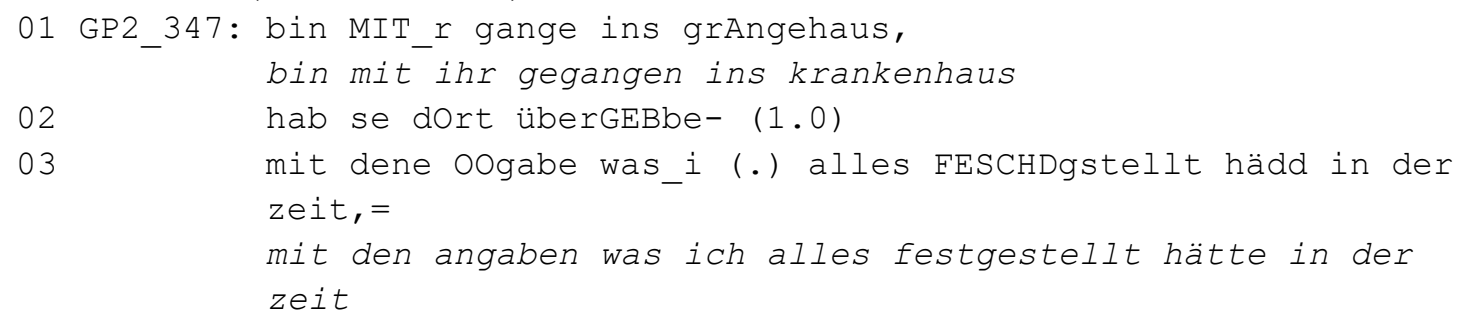

${ }^{5}$ Badisches Wörterbuch = Ochs et al. (1925ff.), im Folgenden abgekürzt als $B W$.

ISSN 1615-3014 


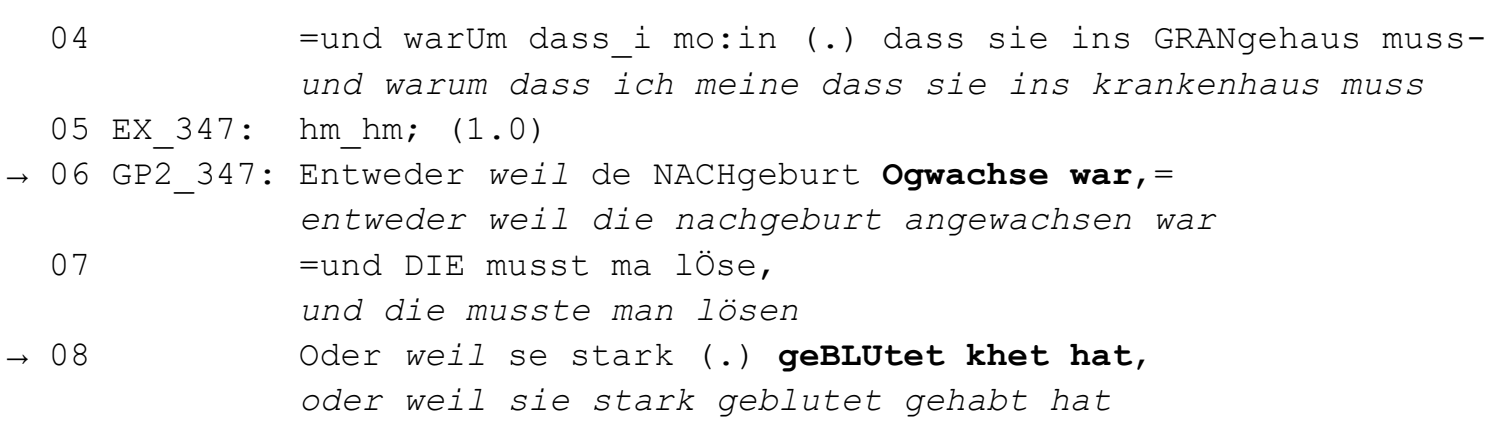

Sie erzählt, es sei in seltenen Fällen vorgekommen, dass sie die schwangeren Frauen ins Krankenhaus bringen musste. Die Ursache dafür beschreibt sie in Zeile 06 (weil de NACHgeburt Ogwachse war) beziehungsweise Zeile 08 (weil se stark (.) geBLUtet khet hat). Dort verwendet sie Plusquamperfekt beziehungsweise Doppelperfekt in den von weil eingeleiteten Äußerungen. Es zeigt sich, dass bei diesen konkreten Handlungen (wenn sie auch nicht auf einen Einzelfall bezogen sind) die Ursache zeitlich vor der Wirkung liegt. Während die Wirkungen also im Perfekt (in den Zeilen 01 und 02) formuliert sind, gebraucht die Sprecherin die Tempora der Vorvergangenheit für die Ursachen.

Der Sprecher GP1_1174 erzählt in Beispiel (2) von seinem Vater. Dieser hatte eigentlich Abitur gemacht, musste dann aber die Landwirtschaft seiner Eltern übernehmen, da sein Bruder im Krieg gefallen war.

(2) Kriegsfolgen (Herten: REDI+FLARS)

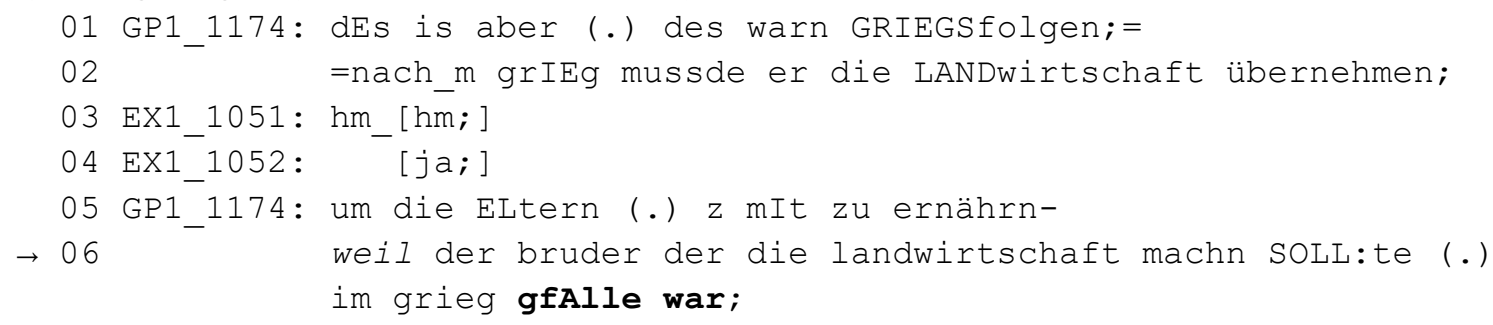

Auch hier gebraucht der Informant in Zeile 06 das Plusquamperfekt (gfAlle war) für die Beschreibung der Ursache. Diese ist zeitlich vor der in Zeile 02 mit Präteritum beschriebenen Situation (mussde er...übernehmen).

Die wichtigsten Indikatoren für die Bestimmung von Vorvergangenheit sind Adverbien. Wie die eben beschriebenen Subjunktionen können sie durch den Ausdruck von Vorzeitigkeit direkt oder durch den Ausdruck von Nachzeitigkeit beim Bezugsverb indirekt auf Vorvergangenheit hindeuten. Die Adverbien scho(n) und vorher indizieren beispielsweise eine Vorvergangenheitsbedeutung des Doppelperfekts und des Plusquamperfekts; die Adverbien no (,dann') und dnooch (,danach') die Nachzeitigkeitsbedeutung des Bezugstempus.

In den Beispielen (3-5) weisen die Adverbien scho(n) und vorher auf die Vorvergangenheitsbedeutung des Doppelperfekts hin. In Beispiel (3) erzählt die Gewährsperson GP1_237, dass ihre Familie fast nichts zu essen gehabt habe, während ihr Mann im Krieg war.

(3) Im Krieg gestorben (Hauingen SSA)

01 GP1_237: no nOch_m CHRIAG, (.)

dann nach dem krieg

02

no het a in CHRIAG mIassa nomal,

dann hat er in (den) krieg gemusst nochmal 


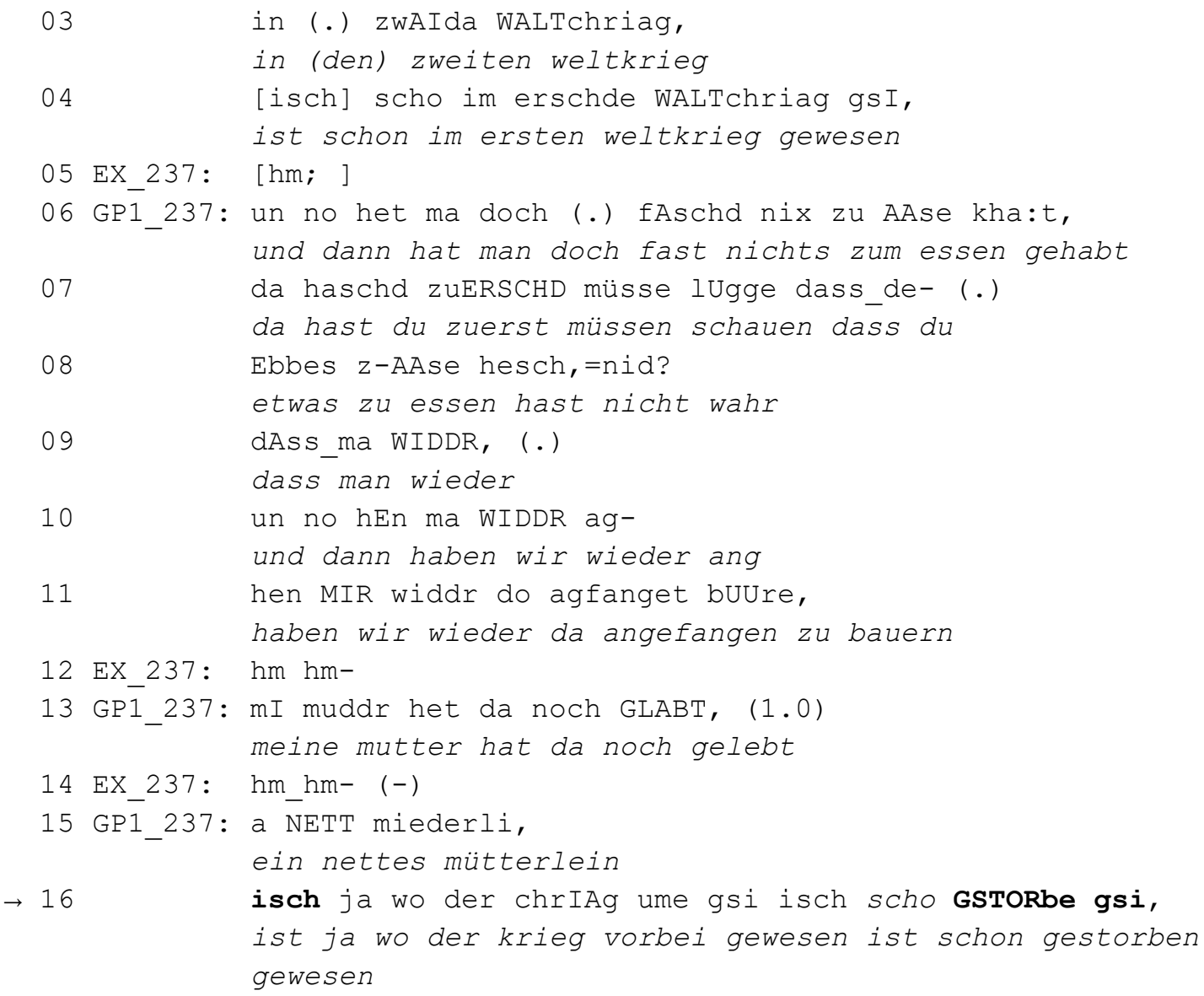

In den Zeilen 01 bis 04 berichtet die Sprecherin, dass ihr Mann nach dem ersten Weltkrieg im zweiten Weltkrieg erneut als Soldat eingezogen wurde. Daraufhin habe ihre Familie Schwierigkeiten gehabt, genug zu essen zu bekommen (Z. 06-09). Deshalb hätten sie erneut Landwirtschaft betreiben müssen (Z. 11). Zu diesem Zeitpunkt habe die Mutter der Gewährsperson noch gelebt (Z. 13). Nach dem Krieg sei sie allerdings schon gestorben gewesen (Z. 16). Das Adverb scho indiziert hierbei die Doppelperfektkonstruktion (isch...GSTORbe gsi) als vorzeitig zur in der Vergangenheit situierten Verbalhandlung (wo der chrIAg ume gsi isch). Somit wird die Vorvergangenheitsbedeutung der Doppelperfektkonstruktion deutlich.

Auch in Beispiel (4) deutet das Adverb scho auf die Vorvergangenheitsbedeutung des Doppelperfekts hin. Hier erzählt der Sprecher GP1_1082 von einem Besuch bei seinen Eltern zum Essen.

(4) Essen (Holzen REDI+FLARS)

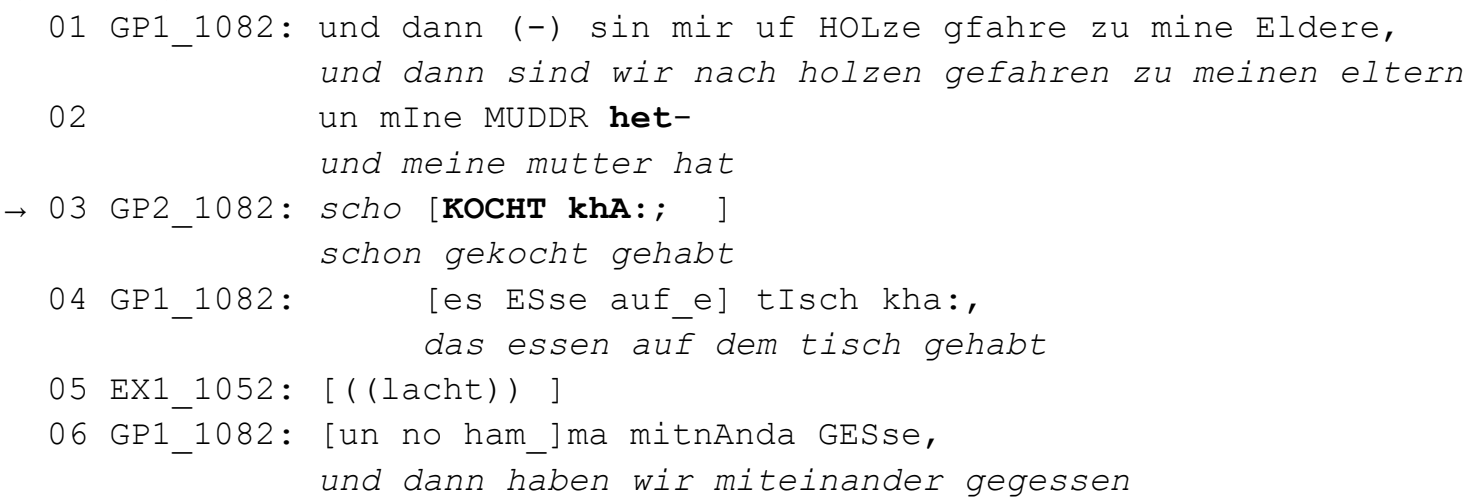


In den Zeilen 02-04 berichtet GP1_1082, dass das Essen auf dem Tisch gestanden habe, als sie dort angekommen seien. GP2_1082 ergänzt simultan die Doppelperfektkonstruktion scho KOCHT khA: (Z. 03). Auch bei dieser Ko-Konstruktion wird die Vorvergangenheitsbedeutung des Doppelperfekts ersichtlich. Das Essen stand auf dem Tisch, als die Gewährspersonen in Holzen angekommen sind. Die Mutter muss demnach schon davor gekocht haben.

Genauso verhält es sich in Beispiel (5). Hier markiert das Adverb vorher die Vorvergangenheitsbedeutung des Doppelperfekts. Der Sprecher GP1_985 erzählt in diesem Beispiel von einem Bild in seiner Wohnung, das seinen Hof zeigt.

(5) Abmalen (Furtwangen SSA)

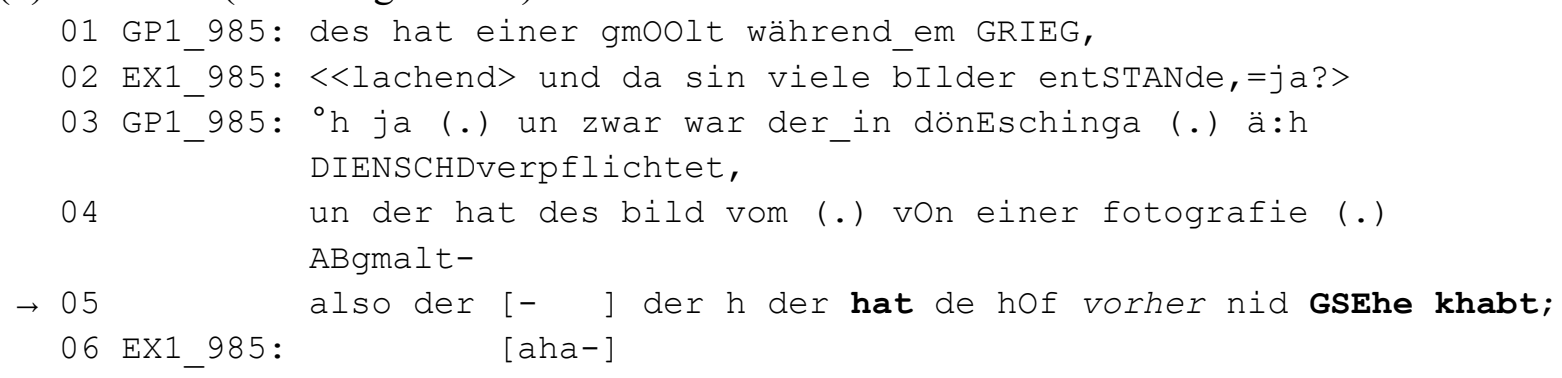

Der Informant berichtet, ein Mann habe dieses Bild während des Kriegs gemalt (Z. 01). Allerdings habe dieser Mann den Hof selbst nicht gesehen, sondern von einer Fotografie abgemalt (Z. 04-05). Die Vorvergangenheitsbedeutung des Doppelperfekts in Zeile 05 (hat...GSEhe $k h a b t)$ wird hier durch das Adverb vorher (Z. 05) ersichtlich.

Adverbien können auch indirekt die Vorvergangenheitsbedeutung von Doppelperfekt und Plusquamperfekt indizieren, indem sie das Kontrasttempus (und damit die Referenzzeit) als nachzeitig darstellen. Vor allem die Adverbien no (,dann') und dnooch (,danach') markieren die Nachzeitigkeitsbedeutung des Bezugstempus.

In Beispiel (6) erklärt die Gewährsperson GP1_619, was unter einem BARre (,Barren') (Z. 02) $\mathrm{zu}$ verstehen ist.

(6) Barren (Greffern SSA)

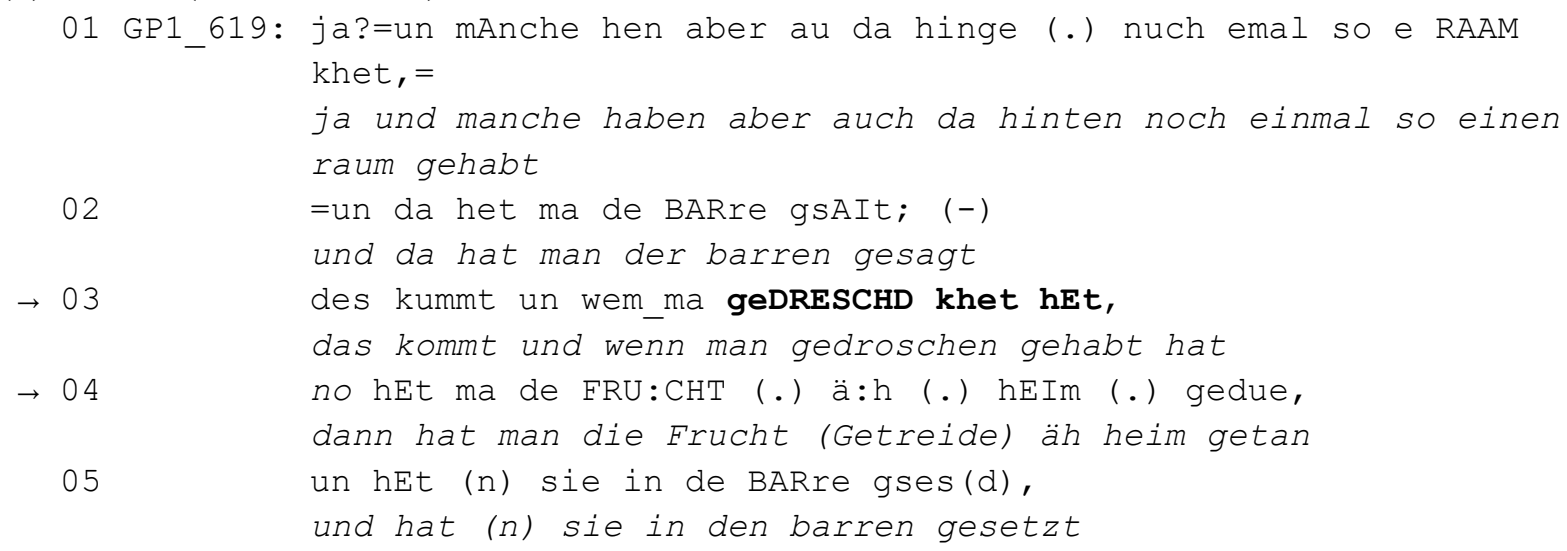

GP1_619 erzählt in den Zeilen 01 und 02, ein Barren sei ein zusätzlicher Gebäudeteil mancher Bauernhöfe. In diesen sei das gedroschene Getreide gebracht worden (Z. 03-05). Die Vorvergangenheitsbedeutung des Doppelperfekts in Zeile 03 (geDRESCHD khet hEt) wird im Kontrast mit der Vergangenheitsbedeutung des Perfekts in Zeile 04 (hEt...hEIm (.) gedue) deutlich. 
Die temporale Subjunktion wenn in Zeile 03 gibt keinen Hinweis darauf, ob die zugehörige Verbalhandlung gleichzeitig oder vorzeitig zur Referenzzeit des übergeordneten Satzes anzusehen ist. Erst das Adverb no (,dann') in Zeile 04 verdeutlicht die Nachzeitigkeitsbedeutung des Perfekts und damit die Vorvergangenheitsbedeutung des Doppelperfekts. Zusätzlich ergibt sich logischerweise, dass Getreide zunächst gedroschen werden muss (Z. 03), ehe es nach Hause gebracht werden kann (Z. 04). Eine Lesart der beiden Verbalhandlungen als gleichzeitig wäre hierbei unsinnig. Neben den eben beschriebenen expliziten Hinweisen auf Vorvergangenheit können auch kontextuelle Hinweise ausgemacht werden. In den Beispielen (7-9) lässt sich die Vorvergangenheitsbedeutung des Plusquamperfekts durch Verbsemantik und den Kontext bzw. das Weltwissen erschließen.

Hier (7) erzählt die Sprecherin GP5_644 vom Ablauf der Hochzeitsveranstaltungen in ihrem Ort.

(7) Patenonkel (Liel SSA)

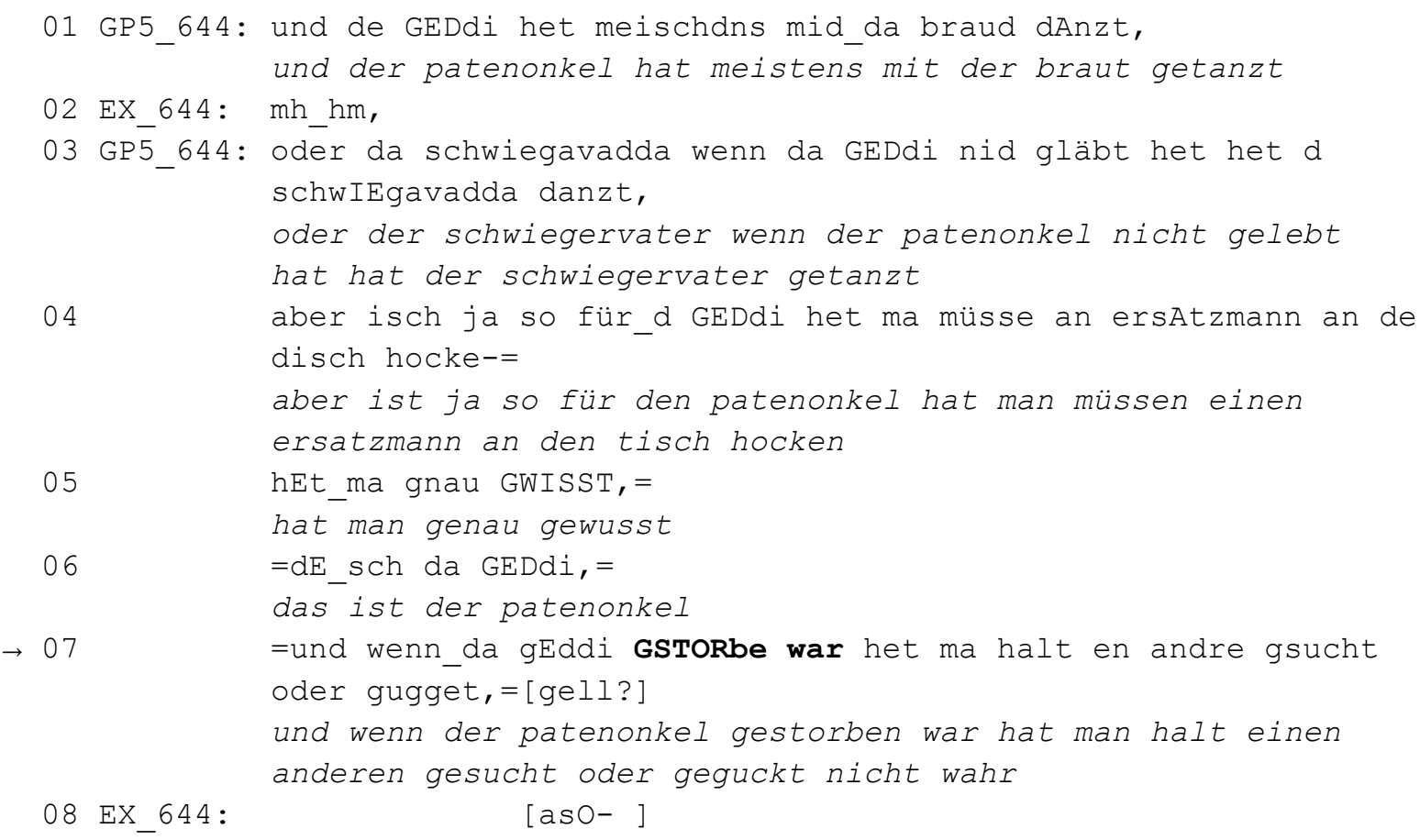

Bei Hochzeiten sei es üblich gewesen, dass der Patenonkel mit der Braut tanzte (Z. 01). Wenn dieser nicht mehr lebte, habe stattdessen der Schwiegervater mit der Braut getanzt (Z. 03). An den Tischplatz des verstorbenen Patenonkels hätte man dann einen Ersatzmann gesetzt (Z. 04), den man nach dem Tod des Patenonkels gesucht habe (Z. 07). Die Vorvergangenheitsbedeutung des Plusquamperfekts in Zeile 07 ergibt sich hier durch eine Kombination aus Verbsemantik und Kontext. Aus dem in Zeile 04 gesetzten Kontext wird ersichtlich, dass es sich beim Ersatz-Suchen um eine Folge des Sterbens des Patenonkels handelt. Dementsprechend liegt das in Zeile 07 beschriebene Ereignis des Sterbens zeitlich vor dem in der Vergangenheit liegenden Ereignis des Ersatz-Suchens.

Auch in Beispiel (8) ist die Vorvergangenheitsbedeutung des Doppelperfekts durch Verbsemantik und Kontext ersichtlich. Hier fragt die Exploratorin EX1_993 den Sprecher GP1_993 nach dem dialektalen Wort für Weide (Z. 1). 
(8) Weide (Kuppenheim SSA)

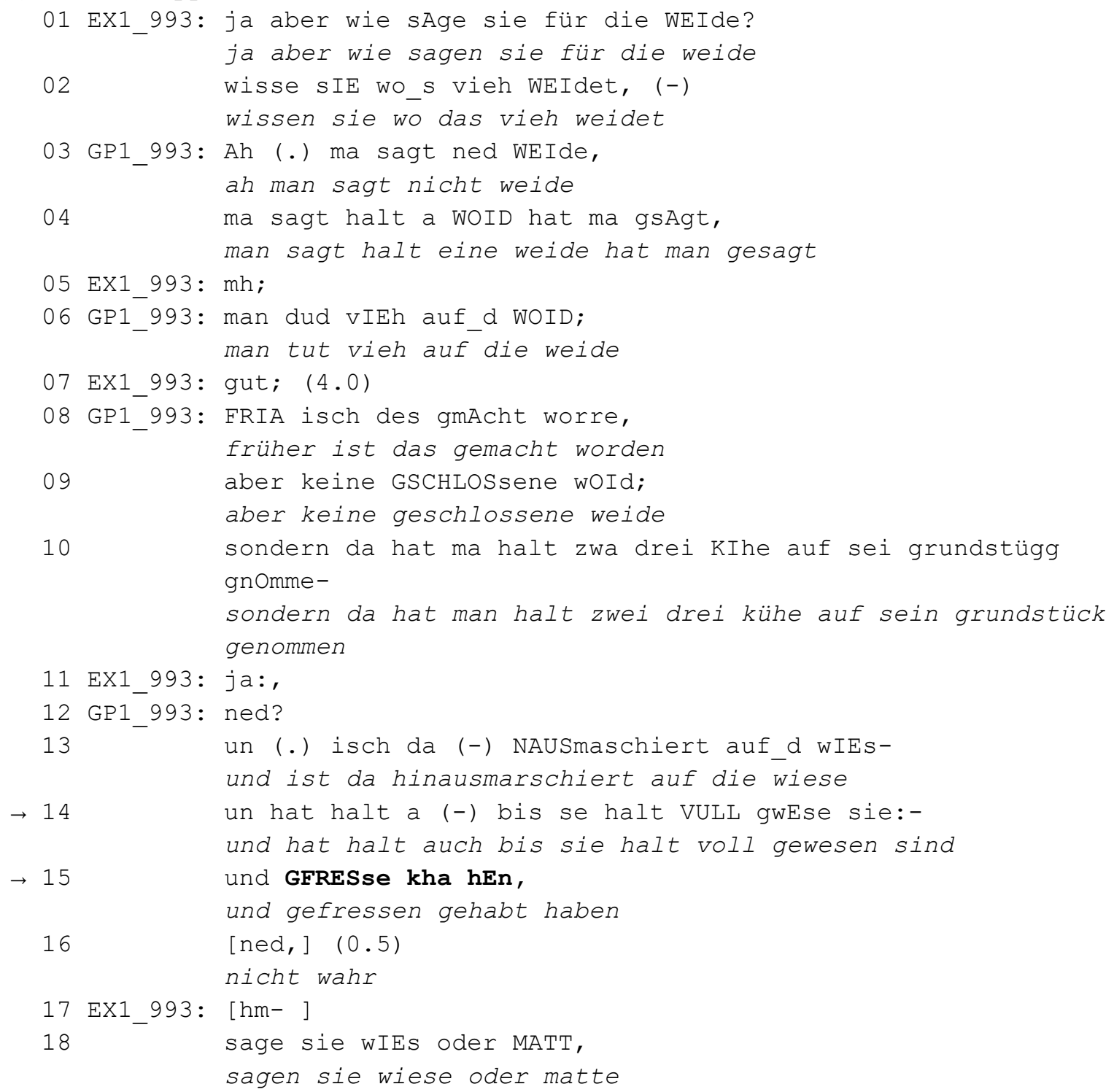

GP1_993 beantwortet die Frage nach dem Wort Weide in den Zeilen 03-04 mit WOID und expandiert seine Antwort in Zeile 06 durch ein Beispiel. Die Beantwortungssequenz ist mit dieser Antwort und der Responsivpartikel gut (Z. 07) der Exploratorin abgeschlossen. Das wird auch durch die anschließende viersekündige Pause (Z.07) deutlich. Daraufhin beginnt GP1_993 in Zeile 08 eine Erzählung darüber, wie Weiden früher beschaffen waren. Dabei habe es sich nicht um geschlossene Weiden gehandelt, sondern um Flächen, die für alle frei zugänglich waren und auf die man seine Kühe geführt habe (Z. 09-13). Dort seien diese geblieben, bis sie voll gewesen seien (Z. 14). Diese Sequenz ist aufgrund der vorangegangenen langen Pause und der damit verbundenen Beendigung der vorherigen Abfragesequenz als Spontansprache zu betrachten. Die Vorvergangenheitsbedeutung des Doppelperfekts in Zeile 15 (GFRESse kha $h E n)$ wird durch die Semantik des Verbs fressen und den Kontext (Z. 14) deutlich. Die Konjunktion und (Z. 15) markiert die Referenzzeit der beiden Verbalhandlungen als gleichzeitig. Da es sich bei der Verbalhandlung des Voll-Seins um das logische Ergebnis des Fressens handelt, liegt die Ereigniszeit der Doppelperfektkonstruktion (Z. 15) zeitlich vor der Ereigniszeit der Perfektkonstruktion (bis se halt VULL gwEse sie:). Da letztere in der Vergangenheit ist, 
drückt das Doppelperfekt hier also Vorvergangenheit aus. Das heißt, bei der Perfektkonstruktion wird eine Referenzzeit in der Vergangenheit gesetzt, zu der die Ereigniszeit gleichzeitig ist; bei der Doppelperfektkonstruktion wird eine zeitgleiche Referenzzeit gesetzt, zu der die Ereigniszeit vorzeitig (und damit vorvergangen) ist. Damit erfüllt das Doppelperfekt in diesem Beispiel den in 3.1. vorgestellten zweifachen Verweis eines Tempus der Vorvergangenheit.

In Beispiel (9) ist die Vorvergangenheitsbedeutung des Plusquamperfekts durch Weltwissen und den Kontext ersichtlich. Hier erzählt der Sprecher GP1_1056, dass er früher Spinnräder gebaut habe.

(9) Schneller als geplant (St. Peter REDI+FLARS)

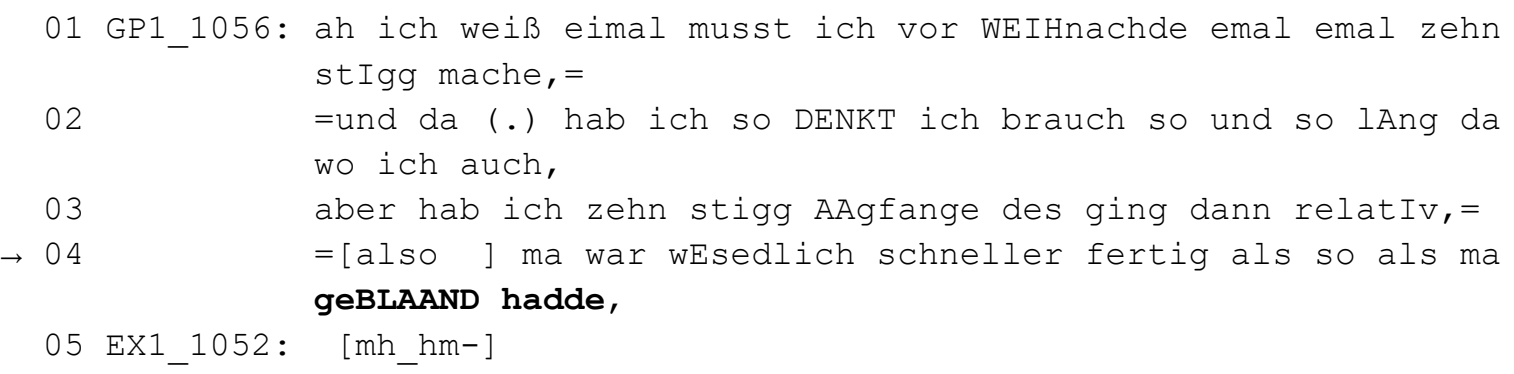

GP1_1056 berichtet, er habe einmal vor Weihnachten zehn Spinnräder anfertigen müssen (Z. 01). Die Vorvergangenheitsbedeutung des Plusquamperfekts in Zeile 04 (geBLAAND hadde) ergibt sich sowohl durch den Kontext als auch durch Weltwissen. Zum einen erwähnt GP1_1056 in Zeile 02, dass er nach dem Erhalt des Auftrags versuchte einzuschätzen, wie lange er brauchen werde. Daraufhin habe er mit seiner Arbeit angefangen (Z. 03) und diese schneller beendet als geplant (Z. 04). Die Verbalhandlung des Planens schildert dieselbe Situation, die bereits in Zeile 02 beschrieben wurde. Dementsprechend ist sie zeitlich vor Beginn bzw. Beendigung der Arbeit. Zum anderen ergibt sich durch das Weltwissen, dass eine Zeitkalkulation (geBLAAND hadde in Zeile 04) immer vor Beendigung der Arbeit (war...schneller fertig in Zeile 04) geschieht.

\subsection{Quantitative Verteilung der Vorvergangenheitsbedeutung}

Das Untersuchungskorpus enthält insgesamt 284 Doppelperfekt- und Plusquamperfekt-Token im Indikativ. Lediglich zwei Doppelperfekt-Belege sind im Konjunktiv I. Diese wurden, wie eingangs beschrieben, in dieser Analyse nicht berücksichtigt. Dennoch lässt sich erkennen, dass das Doppelperfekt im Hoch- und Oberrheinalemannischen deutlich seltener im Konjunktiv I gebraucht wird als in der geschriebenen überregionalen Sprache (cf. Hundt 2011: 23).

Einige Belege mussten aufgrund von schlechter Tonqualität der Aufnahmen ausgeschlossen werden. Teilweise konnte so ein Token nicht zweifelsfrei als Plusquamperfekt beziehungsweise Doppelperfekt eingeordnet werden. In Fällen, in denen das Doppelperfekt beziehungsweise Plusquamperfekt eindeutig als solches identifiziert werden konnte, verhinderten manchmal Bandabbrüche oder Tonstörungen eine ausreichende Analyse des Gesprächskontexts. Zudem konnte trotz der oben beschriebenen Kriterien in manchen Fällen nicht zweifelsfrei entschieden werden, ob das Tempus eine Vergangenheits- oder eine Vorvergangenheitsbedeutung hat. Solche Belege sind daher im Folgenden als Zweifelsfälle gelistet. 
Die quantitative Auswertung zeigt eine höhere Frequenz des Doppelperfekts. 217 von 284 Token entfallen auf das Doppelperfekt (76,4\%), 67 Token (23,6\%) auf das Plusquamperfekt (siehe Tabelle 1).

\begin{tabular}{|l|l|l|}
\hline Tempus & absolute Häufigkeit & relative Häufigkeit \\
\hline Doppelperfekt & 217 & $76,4 \%$ \\
\hline Plusquamperfekt & 67 & $23,6 \%$ \\
\hline Gesamt & 284 & $100 \%$ \\
\hline
\end{tabular}

Tabelle 1: Absolute und relative Häufigkeiten von Doppelperfekt und Plusquamperfekt im Gesamtkorpus

Wie Tabelle 2 zeigt, drücken Doppelperfekt und Plusquamperfekt in den meisten Fällen (85,3\% bzw. 86,6\%) Vorvergangenheit aus.

\begin{tabular}{|l|l|l|l|l|}
\hline & Vergangenheit & $\begin{array}{l}\text { Vorvergangen- } \\
\text { heit }\end{array}$ & Zweifelsfälle & Gesamt \\
\hline Doppelperfekt & $16(7,4 \%)$ & $185(85,3 \%)$ & $16(7,4 \%)$ & $217(100 \%)$ \\
\hline $\begin{array}{l}\text { Plusquam- } \\
\text { perfekt }\end{array}$ & $2(3,0 \%)$ & $58(86,6 \%)$ & $7(10,4 \%)$ & $67(100 \%)$ \\
\hline Gesamt & $18(6,3 \%)$ & $243(85,6 \%)$ & $23(8,1 \%)$ & $284(100 \%)$ \\
\hline
\end{tabular}

Tabelle 2: Absolute und relative Häufigkeiten in der Verwendung von Doppelperfekt und Plusquamperfekt als Ausdruck einfacher Vergangenheit und Vorvergangenheit im Gesamtkorpus

Ein Chi-Quadrat-Test ergibt für den Vergleich der Vorvergangenheitsbedeutung der beiden Formen einen nicht-signifikanten Wert. Doppelperfekt und Plusquamperfekt unterscheiden sich hinsichtlich ihrer Vorvergangenheitsbedeutung also nicht voneinander und können daher als Tempora der Vorvergangenheit angesehen werden.

Betrachtet man die beiden Teilkorpora (SSA und REDI+FLARS), so zeigen sich marginale Unterschiede in der Vergangenheits- und Vorvergangenheitsbedeutung. Die nachfolgende Tabelle 3 präsentiert diese Verteilung in den Korpora SSA und REDI+FLARS.

\begin{tabular}{|l|l|l|l|l|}
\hline & Vergangenheit & $\begin{array}{l}\text { Vorvergangen- } \\
\text { heit }\end{array}$ & Zweifelsfälle & Gesamt \\
\hline SSA & \multicolumn{5}{|l|}{} \\
\hline Doppelperfekt & $6(4,5 \%)$ & $115(87,1 \%)$ & $11(8,3 \%)$ & $132(100 \%)$ \\
\hline $\begin{array}{l}\text { Plusquam- } \\
\text { perfekt }\end{array}$ & $2(8,0 \%)$ & $20(80,0 \%)$ & $3(12,0 \%)$ & $25(100 \%)$ \\
\hline Gesamt & $8(5,1 \%)$ & $135(86,0 \%)$ & $14(8,9 \%)$ & $157(100 \%)$ \\
\hline \multicolumn{5}{|l|}{} \\
\hline REDI+FLARS & $10(11,8 \%)$ & $70(82,4 \%)$ & $5(5,9 \%)$ & $85(100 \%)$ \\
\hline Doppelperfekt & $0(0 \%)$ & $38(90,5 \%)$ & $4(9,5 \%)$ & $42(100 \%)$ \\
\hline $\begin{array}{l}\text { Plusquam- } \\
\text { perfekt }\end{array}$ & $10(7,9 \%)$ & $108(85,0 \%)$ & $9(7,1 \%)$ & $127(100 \%)$ \\
\hline Gesamt
\end{tabular}

Tabelle 3: Absolute und relative Häufigkeiten in der Verwendung von Doppelperfekt und Plusquamperfekt als Ausdruck einfacher Vergangenheit und Vorvergangenheit in den beiden Teilkorpora 
In beiden Teilkorpora überwiegt sowohl beim Doppelperfekt als auch beim Plusquamperfekt die Vorvergangenheitsbedeutung. Trotz der höheren Belegzahl in REDI+FLARS im Vergleich zum SSA kommt das Plusquamperfekt in ersterem Korpus kein Mal in der Vergangenheitsbedeutung vor. Im Korpus SSA drückt das Doppelperfekt mit 87,1\% etwas häufiger Vorvergangenheit aus als das Plusquamperfekt mit 80,0\%. Im Korpus REDI+FLARS besitzt das Doppelperfekt dagegen mit 82,4\% weniger häufig Vorvergangenheitsbedeutung als das Plusquamperfekt mit 90,5\%. Um zu überprüfen, ob diese Unterschiede signifikant sind, habe ich eine binär logistische Regressionsanalyse für den Vergleich der beiden Korpora und der Vorvergangenheitsbedeutung von Doppelperfekt und Plusquamperfekt durchgeführt. Mit der Regressionsanalyse kann der Einfluss unabhängiger Variablen auf eine abhängige gemessen werden. Die unabhängigen Variablen sind hier Ereigniszeit (i. e. Vergangenheits- oder Vorvergangenheitsbedeutung) und Korpus (i. e. SSA oder REDI+FLARS); die abhängige Variable ist das gewählte Tempus (d. h. Doppelperfekt oder Plusquamperfekt). Diese Regressionsanalyse ergab einen nicht-signifikanten Wert für den Einfluss der beiden unabhängigen Variablen auf die abhängige. Das heißt, hinsichtlich der Vorvergangenheitsbedeutung gibt es sowohl synchron als auch diachron keinen statistisch signifikanten Unterschied zwischen den beiden Tempora.

Es lässt sich daher festhalten, dass Doppelperfekt und Plusquamperfekt mit dem Ausdruck von Vorvergangenheit dieselbe Bedeutung besitzen. Diachron hat sich diese Bedeutung bei beiden Tempora statistisch nicht-signifikant verändert.

\section{$4 \quad$ Sprachwandel}

Wir haben gesehen, dass sich Doppelperfekt und Plusquamperfekt hinsichtlich ihrer Vorvergangenheitsbedeutung synchron und diachron nicht unterscheiden. Es stellt sich nun die Frage, ob die Koexistenz dieser bedeutungsgleichen Tempora Gegenstand eines Sprachwandels ist. Zur Untersuchung eines möglichen Sprachwandels vergleiche ich die beiden Teilkorpora hinsichtlich ihres Verhältnisses von Doppelperfekt und Plusquamperfekt. Zusätzlich betrachte ich die Verteilung der Hilfsverben haben und sein zur Tempusbildung, um zu überprüfen, ob Doppelperfekt und Plusquamperfekt mit beiden Hilfsverben gebildet werden können und ob diachron Bildungsbeschränkungen zu sehen sind. Die nachfolgende Tabelle 4 zeigt die absolute und relative Anzahl aller Doppelperfekt- und Plusquamperfekt-Belege in den beiden Teilkorpora.

\begin{tabular}{|l|l|l|l|l|}
\hline & Jahr & Doppelperfekt & Plusquamperfekt & Gesamt \\
\hline SSA & $1974-1985$ & $132(84,1 \%)$ & $25(15,9 \%)$ & $157(100 \%)$ \\
\hline REDI+FLARS & $2007-2013$ & $85(66,9 \%)$ & $42(33,1 \%)$ & $127(100 \%)$ \\
\hline Gesamt & $1974-2013$ & $217(76,4 \%)$ & $67(23,6 \%)$ & $284(100 \%)$ \\
\hline
\end{tabular}

Tabelle 4: Absolute und relative Häufigkeiten von Doppelperfekt und Plusquamperfekt in den beidenTeilkorpora

In beiden Korpora kommen viel mehr Doppelperfekt- als Plusquamperfekt-Belege vor. Dennoch ist vom SSA zu REDI+FLARS ein im Vergleich mit dem Plusquamperfekt deutlicher Rückgang des Doppelperfekts zu beobachten. Während im älteren Korpus noch in $84,1 \%$ das Doppelperfekt und in 15,9\% das Plusquamperfekt genutzt wird, sind es im jüngeren Korpus in $66,9 \%$ Doppelperfekt und in 33,1\% Plusquamperfekt. 
Ein Chi-Quadrat-Test ergibt einen Wert von 11,5***; Cramers Phi zeigt mit einem Wert von $\varphi=0,201^{* * *}$ eine schwache Assoziationsstärke an. Es ist also festzuhalten, dass das Untersuchungskorpus einen Einfluss auf die Wahl des Vorvergangenheitstempus hat. Es lässt sich daher ein Sprachwandel statistisch nachweisen, bei dem die Frequenz des Plusquamperfekts gegenüber der des Doppelperfekts zunimmt.

Graphisch lässt sich die eben beschriebene Verteilung von Doppelperfekt und Plusquamperfekt in den beiden Teilkorpora wie in Graphik 1 darstellen. Der orangene Balken zeigt den prozentualen Anteil der Plusquamperfekt-Token und der blaue den Anteil der Doppelperfekt-Formen im jeweiligen Teilkorpus.

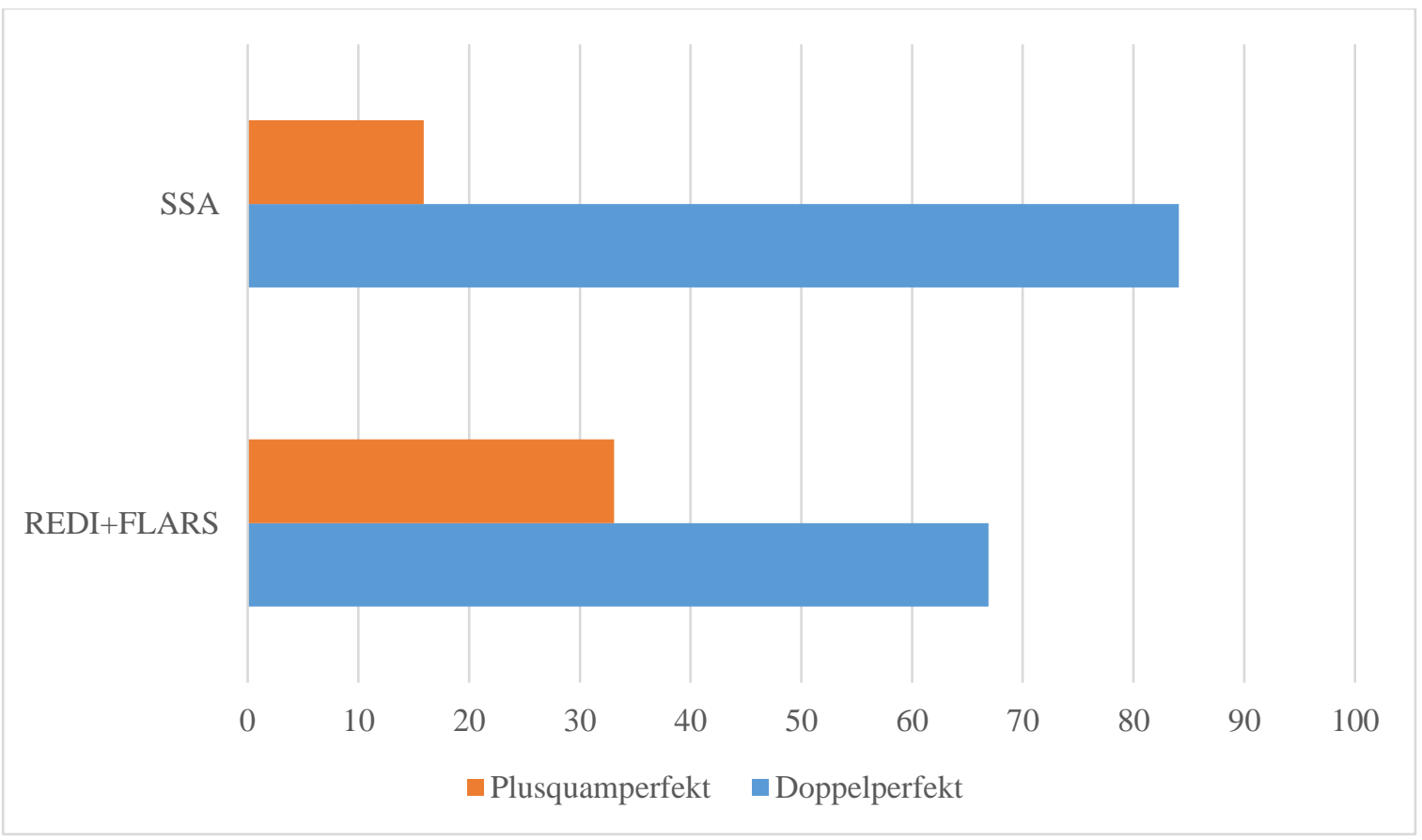

Graphik 1: Absolute und relative Häufigkeiten von Doppelperfekt und Plusquamperfekt in den beiden Teilkorpora

Auch beim Blick auf die Hilfsverben, mit denen Doppelperfekt und Plusquamperfekt gebildet werden, zeigen sich Unterschiede. Die nachfolgenden Tabellen 5 und 6 präsentieren die Verteilung der Token nach den Hilfsverben haben und sein zur Tempusbildung.

\begin{tabular}{|l|l|l|l|}
\hline \multicolumn{2}{|l|}{ haben } & sein & Gesamt \\
\hline SSA & $111(84,1 \%)$ & $21(15,9 \%)$ & $132(100 \%)$ \\
\hline Doppelperfekt & $1(4,0 \%)$ & $24(96,0 \%)$ & $25(100 \%)$ \\
\hline Plusquamperfekt & $112(71,3 \%)$ & $45(28,7 \%)$ & $157(100 \%)$ \\
\hline Gesamt & \multicolumn{5}{|l|}{} \\
\hline \multicolumn{5}{|l|}{ REDI+FLARS } & $32(3,5 \%)$ & $85(100 \%)$ \\
\hline Doppelperfekt & $24(57,1 \%)$ & $18(42,9 \%)$ & $42(100 \%)$ \\
\hline Plusquamperfekt & $106(83,5 \%)$ & $21(16,5 \%)$ & $127(100 \%)$ \\
\hline Gesamt &
\end{tabular}

Tabelle 5: Absolute und relative Häufigkeiten in der Verwendung von Doppelperfekt und Plusquamperfekt nach der Verwendung der Hilfsverben sein und haben zur Tempusbildung in den Teilkorpora 
In beiden Korpora wird mit 71,3\% bzw. 83,5\% weitaus häufiger das Hilfsverb haben zur Tempusbildung gebraucht. Der Grund hierfür ist, dass es grundsätzlich mehr haben- als sein-selegierende Verben gibt. Im Korpus SSA sind mit 84,1\% vs. 15,9\% die Mehrzahl der Doppelperfekt-Token mit dem Hilfsverb haben gebildet. Mit 96,0\% sind hingegen fast alle Plusquamperfekt-Belege mit dem Hilfsverb sein gebildet. Im Korpus REDI+FLARS ist das Doppelperfekt mit 96,5\% praktisch auf haben-selegierende Verben beschränkt. Das Plusquamperfekt wird dagegen mit 57,1\% vs. 42,9\% sowohl mit haben-als auch mit sein-selegierenden Verben gebildet. Da sowohl im Standarddeutschen als auch im Alemannischen die meisten Verben das Hilfsverb haben zur Tempusbildung nutzen, ist festzuhalten, dass das Plusquamperfekt im Hoch- und Oberrheinalemannischen im Vergleich zum Standard mehr Token mit Hilfsverb sein aufweist.

\begin{tabular}{|l|l|l|l|}
\hline \multicolumn{2}{|l|}{ haben } & Sein & Gesamt \\
\hline SSA & $111(99,1 \%)$ & $21(46,7 \%)$ & $132(84,1 \%)$ \\
\hline Doppelperfekt & $1(0,90 \%)$ & $24(53,3 \%)$ & $25(15,9 \%)$ \\
\hline Plusquamperfekt & $112(100 \%)$ & $45(100 \%)$ & $157(100 \%)$ \\
\hline Gesamt & \multicolumn{5}{|l|}{} \\
\hline REDI+FLARS & $32(77,4 \%)$ & $18(85,7 \%)$ & $85(66,9 \%)$ \\
\hline Doppelperfekt & $24(22,6 \%)$ & $21(100 \%)$ & $127(100 \%)$ \\
\hline Plusquamperfekt & $106(100 \%)$ &
\end{tabular}

Tabelle 6: Absolute und relative Häufigkeiten der haben- und sein-selegierenden Verben nach der Tempusbildung in den Teilkorpora

Betrachtet man die Verteilung der haben- und sein-selegierenden Verben auf die beiden Tempora (Tabelle 6), fällt auf, dass im Korpus SSA fast alle haben-selegierenden Verben mit Doppelperfekt gebildet werden $(99,1 \%)$. Die sein-selegierenden Verben stehen mit 53,3\% vs. 46,7\% etwas häufiger im Plusquamperfekt. Im Korpus REDI+FLARS dominieren innerhalb der mit Hilfsverb haben gebildeten Tempora das Doppelperfekt, mit 77,4\% vs. 22,6\% allerdings weniger deutlich als im Korpus SSA. Die sein-selegierenden Verben werden mit $18 \mathrm{zu} 3$ Belegen (85,7\% vs. 14,3\%) mehrheitlich im Plusquamperfekt gebraucht.

Die Korrelation zwischen Hilfsverb und Tempus in den beiden Teilkorpora wird durch einen Chi-Quadrat-Test deutlich. Dieser ergibt einen Wert von 76,5***; Cramers-V zeigt mit einem Wert von $0,519^{* * *}$ eine starke Assoziationsstärke an. Damit lässt sich ein Sprachwandel hinsichtlich der Hilfsverben zur Tempusbildung bei Doppelperfekt und Plusquamperfekt statistisch nachweisen.

Graphisch lässt sich die Entwicklung bei den beiden Tempora wie in den beiden Graphiken 2 und 3 darstellen (cf. Tabelle 5). Die orangene Linie zeigt jeweils den prozentualen Anteil der sein-selegierenden Verben und die blaue den Anteil der haben-selegierenden Verben im jeweiligen Teilkorpus. Graphik 2 zeigt die Entwicklung beim Plusquamperfekt, Graphik 3 die Entwicklung beim Doppelperfekt. 


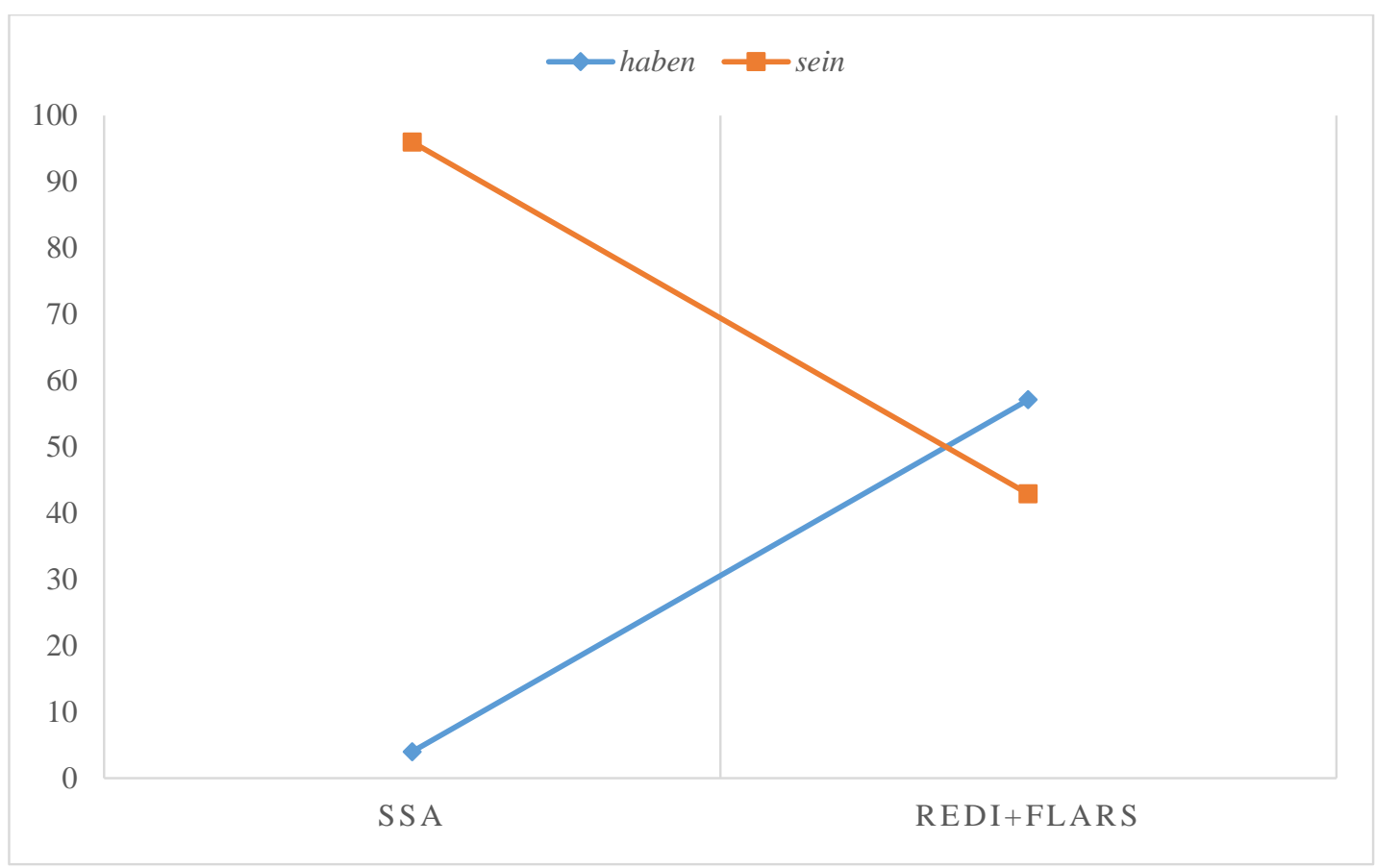

Graphik 2: Relative Häufigkeit der Plusquamperfekt-Token nach der Verwendung der Hilfsverben sein und haben zur Tempusbildung in den beiden Teilkorpora

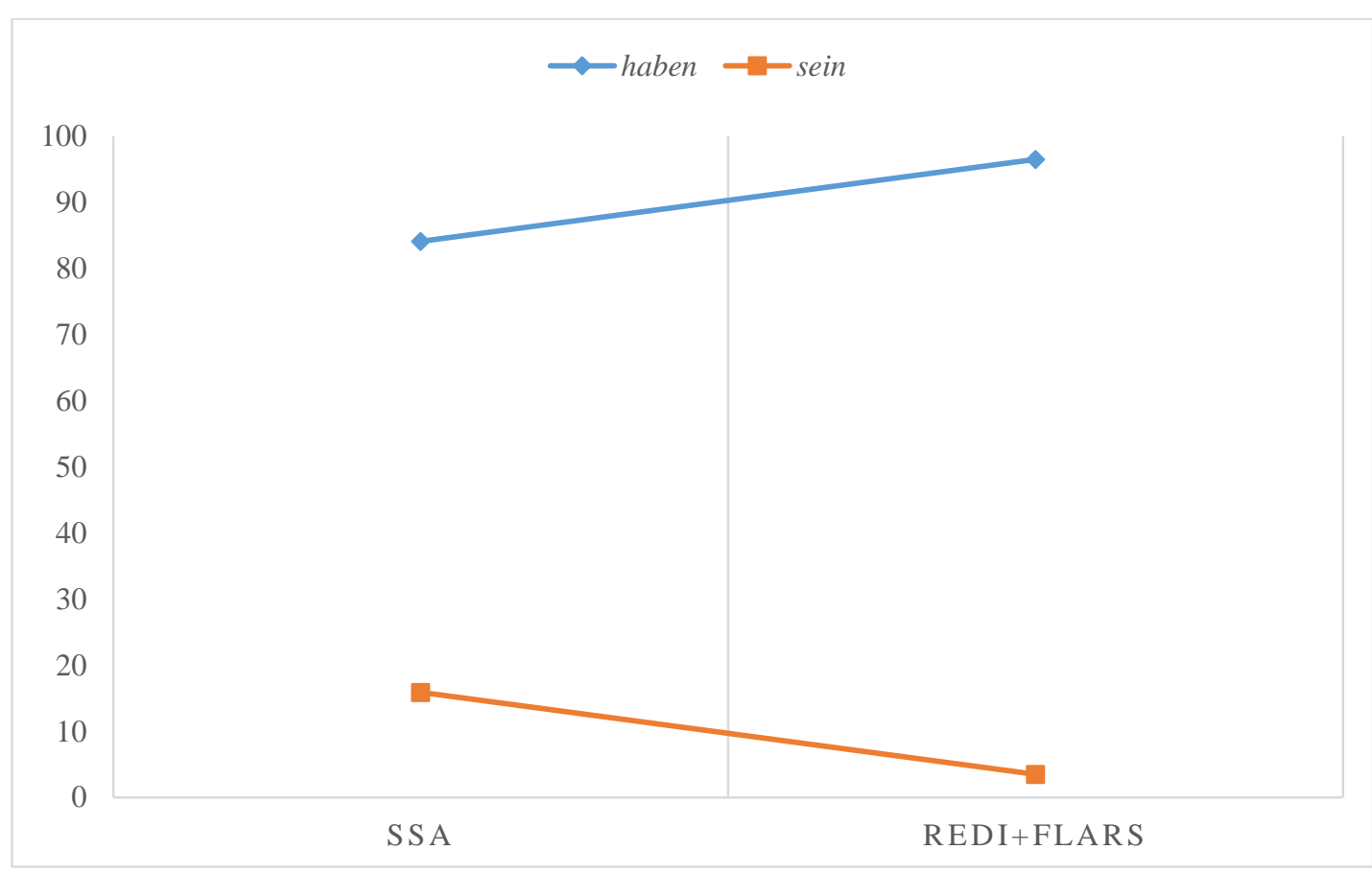

Graphik 3: Relative Häufigkeit der Doppelperfekt-Token nach der Verwendung der Hilfsverben sein und haben zur Tempusbildung in den beiden Teilkorpora

Wir haben gesehen, dass die Frequenz des Plusquamperfekts gegenüber dem Doppelperfekt zwischen dem Korpus SSA und dem Korpus REDI+FLARS deutlich gestiegen ist (siehe Tabelle 4). Darüber hinaus wird das Plusquamperfekt in REDI+FLARS im Gegensatz zum Korpus SSA sowohl mit haben- als auch mit sein-selegierenden Verben gebildet (siehe Graphik 2). Beim Doppelperfekt zeigt sich die umgekehrte Entwicklung (siehe Graphik 3). Während im Korpus SSA sowohl haben- als auch sein-selegierende Verben im Doppelperfekt vorkommen, 
werden in REDI+FLARS fast ausschließlich haben-selegierende Verben im Doppelperfekt gebraucht.

Diachron betrachtet nimmt die Häufigkeit des Plusquamperfekts als Vorvergangenheitstempus zu, die des Doppelperfekts dagegen ab. Hier scheint sich also ein beginnender Sprachwandel abzuzeichnen.

\section{$5 \quad$ Fazit}

Die hier präsentierten Ergebnisse lassen m. E. Rückschlüsse auf das Zusammenwirken von Doppelperfekt und Plusquamperfekt zu. In dieser Arbeit konnte ich zeigen, dass Doppelperfekt und Plusquamperfekt im Hoch- und Oberrheinalemannischen Südwestdeutschlands zu keiner Zeit im Untersuchungszeitraum gleichwertig koexistieren. Das heißt, in den älteren Daten dominiert das Doppelperfekt als Tempus der Vorvergangenheit, indem es sowohl mit haben- als auch mit sein-selegierenden Verben gebildet werden kann; in den jüngeren Daten das Plusquamperfekt (auch, wenn das Doppelperfekt noch immer mit der Mehrzahl der haben-selegierenden Verben gebildet wird und insgesamt häufiger verwendet wird als das Plusquamperfekt). Wir beobachten also möglicherweise den Beginn eines Verdrängungsprozesses, bei dem das Plusquamperfekt das Doppelperfekt ersetzt.

Eine Koexistenz von Doppelperfekt und Plusquamperfekt ist nicht nur im Alemannischen Südwestdeutschlands bzw. im Oberdeutschen zu sehen. Hauser-Suida/Hoppe-Beugel (1972: 254) und Rödel (2007: 267) weisen Doppelperfekt-Belege im gesamten deutschen Sprachraum nach; Şandor (2002: 267) beschreibt die Koexistenz beider Tempora in banatdeutschen Dialekten. Interessant wäre die Frage, ob Doppelperfekt und Plusquamperfekt auch in den anderen Gebieten, in denen die beiden Tempora koexistieren, miteinander konkurrieren. Hundt sieht den süddeutschen Raum zwar als Hauptverbreitungsgebiet des Doppelperfekts an. Allerdings handele es sich lediglich um Unterschiede in der Frequenz, nicht in der Sprachsystematik (cf. Hundt 2011: 12). Viele bisherige Untersuchungen beschränkten sich entweder größtenteils auf Introspektion (beispielsweise Brandner et al. 2016) oder betrachteten unterschiedliche Sprachgebiete ohne Trennung dialektaler und standardnaher Daten miteinander (beispielsweise Rödel 2007). Um Hundts Annahme zu überprüfen, wäre es daher erforderlich, in weiteren Sprachgebieten einen datengestützten Vergleich von Doppelperfekt und Plusquamperfekt zu unternehmen. Aufgrund der eingangs beschriebenen Argumente erscheint ein kausaler Zusammenhang zwischen Wegfall des Plusquamperfekts und historischer Genese des Doppelperfekts unplausibel. Weitere Erkenntnisse zum Zusammenwirken von Doppelperfekt und Plusquamperfekt in einzelnen Gebieten des deutschen Sprachraums könnten aber zur Klärung der Frage beitragen, ob die Frequenz des Doppelperfekts nach dem Ausfall des Plusquamperfekts aufgrund derselben bzw. ähnlichen Bedeutung zugenommen hat. 


\section{Literaturverzeichnis}

Aichinger, Carl Friedrich (1754/1972): Versuch einer teutschen Sprachlehre. Hildesheim/New York: Olms.

Auer, Peter et al. (2015): „Auswirkungen der Staatsgrenze auf die Sprachsituation im Oberrheingebiet (Frontière linguistique au Rhin Supérieur, FLARS)“. In: Kehrein, Roland/Lameli, Alfred/Rabanus, Stefan (eds.): Regionale Variation des Deutschen. Projekte und Perspektiven. Berlin, de Gruyter: 323-348.

Behaghel, Otto (1924): Deutsche Syntax. Eine geschichtliche Darstellung. Band II: Die Wortklassen und Wortformen. Heidelberg: Winter.

Brandner, Ellen et al. (2016): ,Zur Syntax und Semantik des doppelten Perfekts aus alemannischer Sicht“. In: Lenz, Alexandra N./Patocka, Franz (eds.): Syntaktische Variation. Areallinguistische Perspektiven. Göttingen, V\&R: 13-45.

Breuer, Christoph/Dorow, Ralf (1996): Deutsche Tempora der Vorvergangenheit. Trier: Wissenschaftlicher Verlag. (=Fokus. Linguistisch-Philologische Studien 16).

Buchwald, Isabel (2005): ,Zu den temporalen Bedeutungen von Perfekt II und Plusquamperfekt II im gesprochenen Deutsch“. In: Heine, Antje/Hennig, Mathilde/Tschirner, Erwin (eds.): Deutsch als Fremdsprache. Konturen und Perspektiven eines Faches. München, Iudicium: 40-56.

Buchwald-Wargenau, Isabel (2012): Die doppelten Perfektbildungen im Deutschen. Eine diachrone Untersuchung. Berlin/Boston: De Gruyter. (= Studia Linguistica Germanica 115).

Ehrich, Veronika/Vater, Heinz (1989): „Das Perfekt im Dänischen und im Deutschen.“ In: Abraham, Werner/Janssen, Theo (eds.): Tempus - Aspekt - Modus. Die lexikalischen und grammatischen Formen in den germanischen Sprachen. Tübingen, Niemeyer: 103-132. (= Linguistische Arbeiten 237).

Erben, Johannes (1980): Deutsche Grammatik. Ein Abriss. 12. Auflage. Ismaning: Hueber.

Gersbach, Bernhard (1982): Die Vergangenheitstempora in oberdeutscher gesprochener Sprache. Formen, Vorkommen und Funktionen. Untersucht an Tonbandaufnahmen aus BadenWürttemberg, Bayrisch-Schwaben und Vorarlberg. Tübingen: Niemeyer. (= Idiomatica 9).

Hansen-Morath, Sandra (2016): Regionale und soziolinguistische Variation im alemannischen Dreiländereck. Quantitative Studien zum Dialektwandel. Diss. Albert-Ludwigs-Universität Freiburg.

Haß, Norman (2016): Doppelte Zeitformen im Deutschen und im Französischen. Hamburg: Buske. (= Beiträge zur germanistischen Sprachwissenschaft 24).

Hauser-Suida, Ulrike/Hoppe-Beugel, Gabriele (1972): Die Vergangenheitstempora in der deutschen geschriebenen Sprache der Gegenwart. München: Hueber. (= Heutiges Deutsch 1/4).

Hennig, Mathilde (2000): Tempus und Temporalität in geschriebenen und gesprochenen Texten. Tübingen: Niemeyer. (=Linguistische Arbeiten 421).

Hundt, Markus (2011): „Doppelte Perfektkonstruktionen mit haben und sein. Funktionale Gemeinsamkeiten und paradigmatische Unterschiede“. Deutsche Sprache 1/11: 1-24.

Jörg, Ruth (1976): Untersuchungen zum Schwund des Präteritums im Schweizerdeutschen. Bern: Francke. (= Basler Studien zur deutschen Sprache und Literatur 52).

König, Ekkehard (1996): „Kontrastive Grammatik und Typologie". In: Lang, Ewald/Zifonun, Gisela (eds.): Deutsch - Typologisch. Berlin/New York, De Gruyter: 31-54. 
Lindgren, Kaj (1963): „Über Präteritum und Konjunktiv im Oberdeutschen“. Neuphilologische Mitteilungen 64: 264-283.

Litvinov, Viktor P. (1969): „Die doppelte Perfektstreckung im Deutschen“. In: Zeitschrift für Phonetik, Sprachwissenschaft und Kommunikationsforschung 22: 16-24.

Litvinov, Viktor P./Radčenko, Vladimir I. (1998): Doppelte Perfektbildungen in der deutschen Literatursprache. Tübingen: Stauffenburg. (= Studien zur deutschen Grammatik 55).

Maiwald, Cordula (2002): Das temporale System des Mittelbairischen. Synchrone Variation und diachroner Wandel. Heidelberg: Winter. (= Schriften zum bayerischen Sprachatlas 6).

Mattheier, Klaus J. (1994): „Varietätenzensus. Über die Möglichkeiten, die Verbreitung und Verwendung von Sprachvarietäten in Deutschland festzustellen“. In: Mattheier, Klaus J./Wiesinger, Peter (eds.): Dialektologie des Deutschen. Forschungsstand und Entwicklungstendenzen. Tübingen, Niemeyer: 413-442.

Ochs, Ernst et al. (1925ff): Badisches Wörterbuch. Herausgegeben mit Unterstützung des Ministeriums für Wissenschaft, Forschung und Kunst Baden-Württemberg. Vorbereitet von Friedrich Kluge, Alfred Götze, Ludwig Sütterlin, Friedrich Wilhelm, Ernst Ochs. Begonnen von Ernst Ochs, weitergeführt von Karl Friedrich Müller, Gerhard W. Baur, Rudolf Post, bearbeitet von Tobias Streck. Berlin etc.: De Gruyter. 1925-1999 Lahr: Schauenburg, 2000-2012 München: Oldenbourg.

Ölinger, Albert (1574/1975): Underricht der Hoch Teutschen Spraach. Grammatica seu Institutio Verae Germanicae linguae. Hildesheim/New York: Olms. (= Documenta linguistica 5).

Reichenbach, Hans (1947): Elements of symbolic logic. New York: Maximilian.

Rödel, Michael (2007): Doppelte Perfektbildungen und die Organisation von Tempus im Deutschen. Tübingen: Stauffenburg. (= Studien zur deutschen Grammatik 74).

Rowley, Anthony (1983): „Das Präteritum in den heutigen deutschen Dialekten“. Zeitschrift für Dialektologie und Linguistik 50/2: 161-182.

Şandor, Mihaela (2002): „Funktion und Gebrauch der doppelten Perfektformen in den Banater deutschen Mundarten“. Transcarpathica. Germanistisches Jahrbuch. Rumänien 1:253-273.

Schottel, Justus Georg (1641/1967): Ausführliche Arbeit von der teutschen Haubtsprache. Tübingen: Niemeyer.

Semenjuk, Natalija (1981): „Tempus“. In: Guchmann, Mirra/Semenjuk, Natalija (eds.): Zur Ausbildung der Norm der deutschen Literatursprache im Bereich des Verbs (1470-1730). Berlin, Akademie-Verlag: 19-121. (= Zur Ausbildung der Norm der deutschen Literatursprache (1470 - 1730) 5).

SSA = Südwestdeutscher Sprachatlas (1989-2011). Herausgegeben von Steger, Hugo/Gabriel, Eugen/Schupp, Volker. Marburg: Elwert.

Steger, Hugo/Schupp, Volker (eds.) (1993): Einleitung zum Südwestdeutschen Sprachatlas I. Marburg: Elwert.

Stoeckle, Philipp (2014): Subjektive Dialekträume im alemannischen Dreiländereck. Hildesheim: Olms. (= Deutsche Dialektgeographie 112).

Sütterlin, Ludwig (1900): Die deutsche Sprache der Gegenwart. Leipzig: Voigtländer.

Thiel, Rudolf (1964): „Die Zeiten der Vergangenheit““. Sprachpflege 13: 83-85.

Thieroff, Rolf (1992): Das finite Verb im Deutschen. Tempus - Modus - Distanz. Tübingen: Narr. (= Studien zur deutschen Grammatik 40). 
Trier, Jost (1965): „Stilistische Fragen der deutschen Gebrauchsprosa. Perfekt und Imperfekt“. In: Henß, Rudolf/Moser, Hugo (eds.): Germanistik in Forschung und Lehre. Vorträge und Diskussionen des Germanistentages in Essen. 21.-25. Oktober 1964. Berlin, Schmidt: 195-208.

Wilde, Michael (2015): Der Konjunktiv im Schweizerdeutschen. Empirische Studien zu Stabilität und Wandel im deutschen Modussystem. Bern etc.: Lang.

Zybatow, Tatjana (2015): „Ich hab's angekündigt gehabt: Das Doppelperfekt und die Partizipien“. In: Fortmann, Christian/Lübbe, Anja/Rapp, Irene (eds.): Situationsargumente im Nominalbereich. Berlin/Boston, De Gruyter: 259-287.

Zybatow, Tatjana/Weskott, Thomas (2018): „Das Doppelperfekt: Theorie und Empirie“. Zeitschrift für Sprachwissenschaft 37/1: 83-124. 